\title{
Lubrication in cold rolling: elasto-plasto-hydrodynamic lubrication of smooth surfaces
}

\author{
P. M. Lugt, A. W. Wemekamp and W. E. ten Napel \\ University of Twonte, P.O. Box 217, Enschede (The Netherlands) \\ P. van Liempt and J. B. Otten \\ Hoogovens Research Laboratory, P.O. Box 10000, IJmuiden (The Netherlands)
}

(Received October 19, 1992; revised and accepted January 21, 1993)

\begin{abstract}
A model has been developed with respect to hydrodynamic lubrication in cold rolling. The basic model describes the configuration of a rigid, perfectly plastic sheet rolled by a rigid work roll. The governing equations have been solved throughout the complete contact area, i.e. the inlet, the work zone and the outlet zone. Multi-level techniques have been applied to solve these equations together with boundary conditions, resulting in an algorithm solving the problem in $O(n)$ operations. This means that the distribution of the pressure and the traction force in the lubricant film, and the shape of this film, as well as the plastic deformation of the sheet, can be accurately calculated for a large number of nodal points on a minicomputer. Subsequently elastic deformation, work hardening and dynamic behaviour of the flow stress have been incorporated in the model. It will be shown that the influence of these effects on the film thickness or the pressure distribution is considerable.
\end{abstract}

\section{Introduction}

As in most metal forming processes the main reason for lubricating the cold rolling process is to control friction and surface quality. Under most circumstances, steel and aluminium are lubricated in such a way that some asperities will touch and carry part of the load, causing additional friction. Particularly in aluminium foil rolling, where the strip is very thin and the material is relatively soft, the surface quality is fully determined by the lubrication process. If the running surfaces are completely separated by a lubricant film, "hydrodynamic pits" may be formed, resulting in a dull surface, whereas if the lubrication mechanism is of the mixed type, the bright surface of the rolls is printed on the strip.

In this mixed lubrication situation, friction is caused by both viscous shear in the lubricant film and shear between the touching asperities. Low friction reduces the rolling force, which again reduces the elastic flattening of the work rolls and enables a larger reduction. However, if the friction is too low the rolls will skid and the strip will no longer be drawn through the bite.

Figure 1 shows a schematic representation of the cold rolling process. The sheet is subjected to an inlet tension $\sigma_{\mathrm{i}}$ and an outlet tension $\sigma_{\mathrm{o}}$. Owing to symmetry, only half the configuration needs to be considered. The origin of the $x$-coordinate is positioned on the roll centre line. The configuration can be divided into three regions: the inlet zone, the work zone and the outlet zone.

The most widely used analysis describing the plastic deformation process is based on Von Kármán's work [1]. In his analysis of the rolling process the friction is characterized by a Coulomb model. Various other investigators, e.g. Jortner et al. [2], have included roll flattening as well. Furthermore, Cheng [3] and also Bedi and Hillier [4] integrated hydrodynamic lubrication and plasticity. The latter authors minimized the dissipated energy in the work zone in order to solve the pressure distribution etc. However, in their approach, the viscosity was still assumed to be pressure- and temperature-independent. Wilson and Mahdavian [5] and Dow et al. [6] were the first to introduce thermal effects. Tsao and Sargent [7] included mixed lubrication. They used a conventional thermal inlet analysis to determine the inlet film thickness and solved the continuity equation to determine the film thickness in the work zone. Atkins [8] has taken into account the inlet, the work zone and the outlet zone and calculated pointwise elastic deformation and heat development by plastic deformation. 

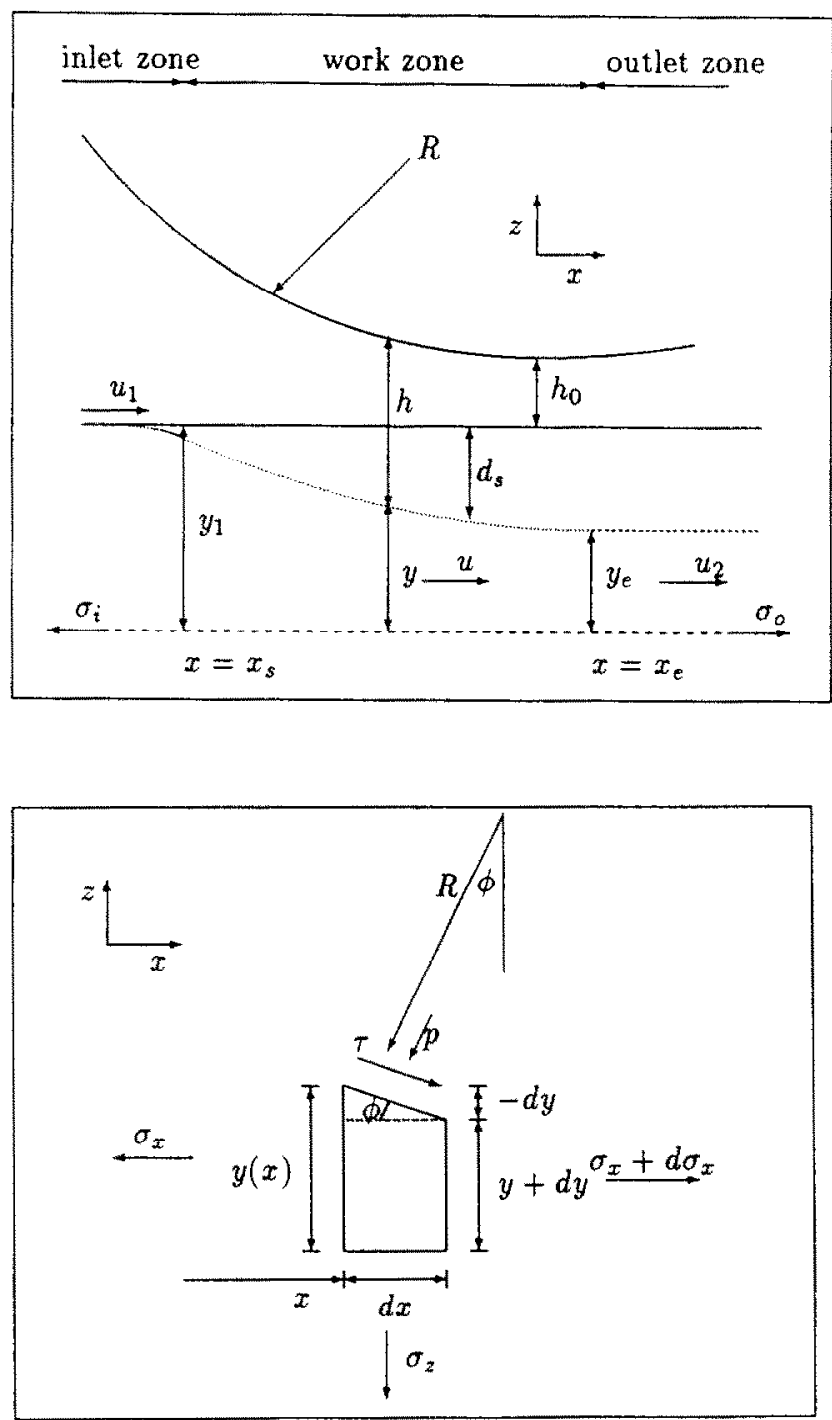

Fig. 1. Representation of the components from the film thickness equation and a slab taken from the sheet.

Although asperity contact is important in analysing the rolling process, research is mainly focused on full film lubrication. The most commonly applied theory is from Wilson and Walowit [9]. They calculated pressure, film thickness and shear stress distribution by applying an analytical solution of the Reynolds equation in the inlet zone and solving the continuity equation in the work zone. Their well known film thickness formula is:

$h_{\mathrm{w}}=\frac{3 \eta_{0} \alpha R\left(u_{1}+u_{\mathrm{r}}\right)}{-x_{\mathrm{s}}\left(1-e^{-\alpha\left(\sigma_{\mathrm{k}}-\sigma_{\mathrm{i}}\right)}\right)}$

Research in this field over the last five years, has been focused on mixed lubrication models, where asperity flattening plays a central role. As mentioned before, Tsao and Sargent [7] developed such a model in 1967. However, only the elastic flattening of the asperities, using the Hertzian theory, was considered.
Sheu [10] was the first to introduce plastic flattening of asperities. He used an averaged Reynolds equation describing the lubricant flow and an upper bound approach for the plastic asperity deformation. Sutcliffe [11] improved this work by applying another deformation mode.

Film thickness always plays a central role in the different lubrication theories. If the film thickness is large, the asperities will not touch and friction will be low. On the other hand, if the film thickness is small, some asperities will touch and friction will be high. In order to calculate the part of the friction determined by asperity contact, the film thickness should be calculated accurately. In this paper it will be shown that, for instance, elastic deformation of the sheet in the inlet will result in film thicknesses significantly larger than found so far. As a result the real area of contact will be much smaller than assumed so far in the mixed lubrication models. This paper describes a basic model calculating pressure, film thickness and shear stress distribution by solving the Reynolds equation in the entire contact area, i.e. the inlet, the work zone and the outlet zone, in combination with plastic deformation using multigrid techniques. Incorporation of various effects, such as non-Newtonian fluid behaviour and elastic deformation of the sheet and roll can then relatively easily be incorporated. In this paper this will be shown for the elastic deformation of the sheet. Moreover, these techniques make it possible to deal with surface defects like bumps, scratches etc.

\section{Equations}

The behaviour of the lubricant between the roll and the sheet can adequately be described by the Reynolds equation [12]:

$\frac{\mathrm{d}}{\mathrm{d} x}\left(\frac{h^{3}}{\eta} \frac{\mathrm{d} p}{\mathrm{~d} x}\right)-12 h \frac{\mathrm{d} u_{\mathrm{a}}}{\mathrm{d} x}-12 u_{\mathrm{a}} \frac{\mathrm{d} h}{\mathrm{~d} x}=0$

where $p$ is pressure, $h$ is film thickness, $\eta$ is dynamic viscosity and $u_{\mathrm{a}}$ is the average velocity. For the cold rolling situation this equation has to be rewritten, since the velocity of the strip surface is not constant. Assuming the metal deformation to be homogeneous, which holds in the case of cold rolling for reductions over $10 \%$ [13], this velocity can be derived using the continuity equation for the strip, $(\mathrm{d} / \mathrm{d} x)(u y)=0$, so the Reynolds equation reads:

$\frac{\mathrm{d}}{\mathrm{d} x}\left(\frac{h^{3}}{\eta} \frac{\mathrm{d} p}{\mathrm{~d} x}\right)+6 u_{1} y_{1} \frac{h}{y^{2}} \frac{\mathrm{d} y}{\mathrm{~d} x}-3\left[2 u_{1} y_{1}(1 / y)+u_{\mathrm{sd}}\right] \frac{\mathrm{d} h}{\mathrm{~d} x}=0$ 
with $u$ for the local strip velocity, $u_{1}$ for the initial strip velocity, $y_{1}$ for half the initial thickness of the strip, $u_{\mathrm{rd}}$ for twice the roll velocity and $y$ for half the strip thickness.

The film shape can be represented by a parabola, approximating the shape of the roll, plus the plastic deformation of the strip $\mathrm{d}_{\mathrm{p}}(x)$ :

$h(x)=h_{0}+\left(x^{2} / 2 R\right)+y_{1}-y(x)$

or

$h(x)=h_{00}+\left(x^{2} / 2 R\right)-y(x)$

with $h_{00}=h_{0}+y_{1}$. Figure 1 shows this schematically.

A large $h_{00}$ corresponds to a large film thickness, which causes a very low pressure in the lubricant film, whereas a decrease of $h_{00}$ results in an increase of pressure. $h_{00}$ is chosen such that the inlet pressure equals the flow stress minus the prescribed value of the inlet tension. As a result the parameter $h_{00}$ describes the vertical displacement of the roll, needed to supply the roll force, the so-called "screw setting".

In the inlet zone, half the sheet thickness $y$ is equal to the inlet thickness $y_{1}$. In the work zone, half the sheet thickness is described by the well-known Von Kármán [1] equation.

$y \frac{\mathrm{d} p}{\mathrm{~d} x}=\sigma_{\mathrm{k}} \frac{\mathrm{d} y}{\mathrm{~d} x}+y \frac{\mathrm{d} \sigma_{\mathrm{k}}}{\mathrm{d} x}+\tau(x)$

With $\tau=(\eta / h)\left[\left(u_{\mathrm{rd}} / 2\right)-u\right]$ (Newtonian fluid), this equation reads:

$y \frac{\mathrm{d} p}{\mathrm{~d} x}=\sigma_{\mathrm{k}} \frac{\mathrm{d} y}{\mathrm{~d} x}+y \frac{\mathrm{d} \sigma_{\mathrm{k}}}{\mathrm{d} x}+\frac{\eta}{2 h}\left[u_{\mathrm{rd}}-2 u_{1} y_{1}(1 / y)\right]$

In the outlet zone the sheet behaves rigidly again.
The relation between $z, \alpha$ and $\eta_{0}$ is:

$z=p_{\mathrm{r}} \alpha /\left[\ln \left(\eta_{0}\right)+9.67\right]$

\section{Boundary conditions}

Since the Reynolds equation predicts negative pressures in a diverging oil film, the so-called cavitation condition has to be fulfilled: $p \geqslant 0$. As a result, the boundary for the pressure in the outlet will become a free boundary: $p=0$ at $x \geqslant x_{\mathrm{c}^{-}}$. In the inlet zone, far away from the gap, the pressure will equal the ambient pressure. So the second boundary for the pressure reads: $x=-\infty, p=0$.

In the inlet zone, half the sheet thickness $y$ is given by $y=y_{1}$.

The boundaries with respect to plastic deformation can be derived using the Von Mises flow criterion together with the vertical force balance on a slab of the sheet: $\sigma_{\mathrm{x}}+p=\sigma_{\mathrm{k}}$. In the inlet and outlet zone the horizontal stress $\sigma_{\mathrm{x}}$ must equal the inlet tension $\sigma_{\mathrm{i}}$ and outlet tension $\sigma_{\mathrm{o}}$ respectively. As a result the plastic or work zone is bounded by:

$x=x_{\mathrm{s}}, \quad p=\sigma_{\mathrm{k}}-\sigma_{\mathrm{i}}$

$x=x_{\mathrm{e}}, \quad p=\sigma_{\mathrm{k}}-\sigma_{\mathrm{o}}$

where $x_{\mathrm{s}}$ and $x_{\mathrm{e}}$ are the start and end of plastic deformation respectively.

At the end of the work zone, plastic deformation will stop and the sheet will become rigid again. Hence the boundary condition regarding the sheet thickness at $x=x_{\mathrm{c}}$ is a Neuman condition reading $x \geqslant x_{\mathrm{c}}, \mathrm{d} y / \mathrm{d} x=0$.

The resulting equations and configurations are too complicated to enable analytical solution. Consequently, the equations will be discretized and solved numerically.

\section{Multigrid techniques}

The viscosity of the lubricant is strongly pressuredependent. The viscosity-pressure relation applied here in the first instance is the Barus [14] equation:

$\eta=\eta_{0} e^{\alpha p}$

Despite the fact that this relation overestimates viscosities for high pressures [15], it will be applied in order to be able to compare the results with those of Wilson and Walowit [9].

In the second part of the paper a more adequate relation, i.e. the Roelands relation [16], will be applied.

$\eta=\eta_{0} \exp \left\{\left(\ln \eta_{0}+9.67\right)\left[\left(1+p / p_{r}\right)^{2}-1\right]\right\}$

with $p_{\mathrm{r}}=1.962 \times 10^{8} \mathrm{~Pa}$.
To obtain an accurate solution and to be sure that surface roughness can be represented on a grid, it will be necessary to calculate all variables on a large number of nodal points. Application of conventional techniques, such as the widely used Newton-Raphson system approach, will require unacceptably large computer power, since the CPU time for this process is proportional to the second power of the number of nodal points (complexity is $\left.O\left(n^{2}\right)\right)$. Therefore the multigrid method is used here in solving the cold rolling equations. The advantage of this method is that the complexity is about $O(n)$. 
This method has been applied to lubrication problems by Lubrecht [17] in solving so called EHL (elastohydrodynamic lubrication) problems. The solver for this problem has been greatly improved by Venner et al. [18]. The method will not be described here, but for a multigrid introduction the reader is referred to Briggs [19] and for a far more extended outline of these techniques to Brandt [20].

\section{Preliminary results}

In Figs. 2(a) and (b) a characteristic solution is plotted. The solution is calculated using 262145 nodal points. The input parameters for this situation are listed in Table 1.

The film thickness is characterized by a sharp decrease in the inlet zone, while at the boundary of the inlet and work zone a sharp edge appears. This is the result of the sudden start of the deformation at this point. Elastic deformation of the sheet, as well as hardening, has not been taken into account yet, as the deformation model describes rigid, perfectly plastic material behaviour, so $\mathrm{d} \sigma_{\mathrm{k}} / \mathrm{d} x=0$.

Owing to the sharp decrease in film thickness, the pressure rise in the inlet zone is restricted to a small area where the pressure rises from zero to $\sigma_{\mathrm{k}}-\sigma_{\mathrm{i}}$.

The work zone is characterized by a slightly decreasing film thickness $h$, a decreasing sheet thickness and a non-constant pressure. In the case of constant surface velocities the film thickness would be expected to be nearly constant, as in EHL situations, since the shear (Couette) flow dominates the pressure (Poiseuille) flow. Because of the increasing sheet velocity in the $x$ direction, however, the film thickness decreases slightly.

The shear stress, shown in Fig. 2(b), is positive near the inlet wedge of the work zone due to backward slip in this region. As the sheet velocity increases in the $x$-direction the slip decreases, resulting in a decreasing shear stress. At the location where the sheet velocity equals the roll velocity, the so called neutral point, the shear stress becomes zero, after which it becomes
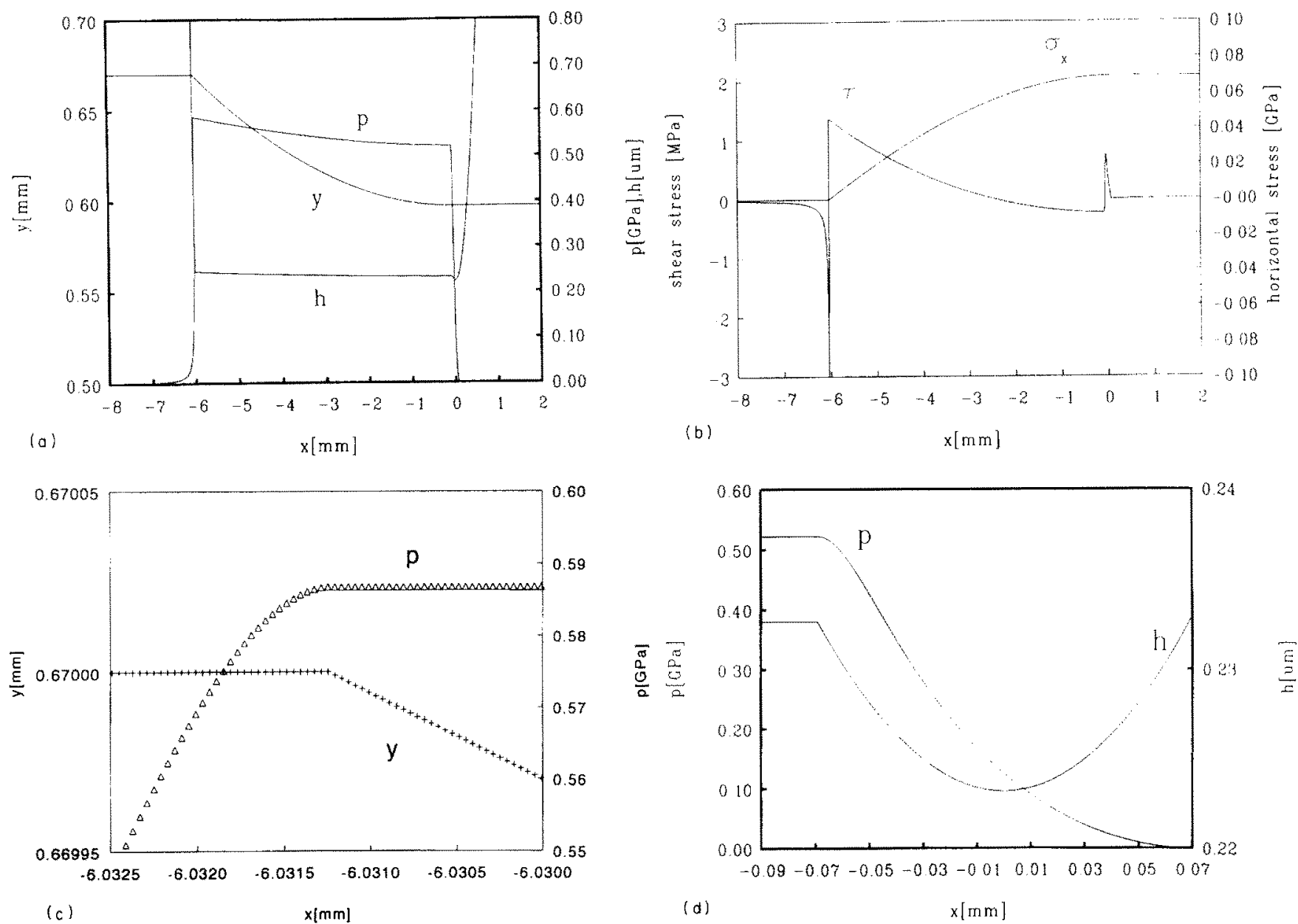

Fig. 2. (a) Characteristic solution of sheet thickness $y$, pressure $p$ and film thickness $h$; (b) characteristic solution of shear stress (working on the sheet) and horizontal stress distribution; (c) pressure and sheet thickness in an area of $2.5 \mu \mathrm{m}$ around $x_{5}$; (d) pressure and film thickness in an area of $0.16 \mathrm{~mm}$ around the line joining roll centres. 
TABLE 1. Some parameters corresponding to the results presented in Fig. 2

\begin{tabular}{lll}
\hline Parameter & Value & Dimension \\
\hline$n$ & 262145 & - \\
$y_{1}$ & 0.670 & $\mathrm{~mm}$ \\
$y_{e}$ & 0.597 & $\mathrm{~mm}$ \\
$h_{\mathrm{i}}$ & 0.246 & $\mu \mathrm{m}$ \\
$h_{\mathrm{w}}$ & 0.251 & $\mu \mathrm{m}$ \\
$h_{\min }$ & 0.223 & $\mu \mathrm{m}$ \\
$R$ & 25.0 & $\mathrm{~cm}$ \\
$\sigma_{\mathrm{k}}$ & 586.8 & $\mathrm{MPa}$ \\
$\sigma_{\mathrm{i}}$ & 0.0 & $\mathrm{MPa}$ \\
$\sigma_{\mathrm{o}}$ & 65.0 & $\mathrm{MPa}$ \\
$p_{\max }$ & 0.587 & $\mathrm{GPa}$ \\
$\tau_{\max }$ & 1.38 & $\mathrm{MPa}$ \\
$\mu_{\max }$ & 0.00235 & - \\
$w$ & 3292.6 & $\mathrm{kN} \mathrm{m}^{-1}$ \\
$x_{\mathrm{s}}$ & -6.03 & $\mathrm{~mm}$ \\
$x_{\mathrm{np}}$ & -2.49 & $\mathrm{~mm}$ \\
$x_{e}$ & -0.069 & $\mathrm{~mm}$ \\
$\eta_{0}$ & 0.02 & $\mathrm{~Pa} \mathrm{~s}$ \\
$\alpha$ & $0.5 \times 10^{-8}$ & $\mathrm{~Pa}$ \\
$u_{\mathrm{rd}}$ & 20.0 & $\mathrm{~m} \mathrm{~s}$ \\
$u_{1}$ & 9.1 & $\mathrm{~m} \mathrm{~s}$ \\
\hline & & \\
\hline
\end{tabular}

negative due to forward slip. The two peaks in the shear stress at the boundaries of the work zone are caused by the relatively large pressure flow due to a large pressure gradient.

In Fig. 2(c), the pressure and sheet thickness near the point where plastic deformation starts $\left(x=x_{s}\right)$ are plotted. The sharp edge in the pressure curve appears to be locally smooth, whereas the edge in the sheet thickness curve is sharp, due to the sudden start of deformation. Of course, the number of points used here is needlessly high for calculating an accurate solution. The large number used is merely intended to illustrate the possibilities of the developed algorithm with respect to future rough surface calculations.

In Fig. 2(d) the area around the line joining roll centres is enlarged. Plastic deformation stops at $x=-0.06884 \mathrm{~mm}$. Beneath the centre of the roll the material has become rigid again. As a result, the film thickness shows a dip in this area allowing a high negative pressure gradient. The figure also shows that the pressure gradient becomes zero when cavitation occurs.

According to the Wilson and Walowit [9] formula (1) the inlet film thickness $h_{w}=0.251 \mu \mathrm{m}$. This is in good agreement with the inlet film thickness calculated here $\left(h_{\mathrm{i}}=0.246 \mu \mathrm{m}\right.$.). This is no surprise, bearing in mind that the film thickness in a concentrated contact is mainly determined by the inlet zone. Wilson and Walowit assumed this inlet wedge to be straight. Moreover, they assumed the pressure gradient at the start of plastic deformation to be zero. The results here
(Figs. 2(a) and (c)) show that both assumptions are appropriate. However, when the shear stress is increased by using an oil with a large pressure-viscosity coefficient $\alpha$, a typical "pressure hill" will be found, making this assumption dubious.

In the following it will be shown that elastic deformation of the sheet and work hardening of the sheet influence the film thickness considerably.

\section{Elastic deformation of the sheet in the inlet zone}

In the following, an elastic-plastic material model will be used while the deformation will be assumed to be uniform over the thickness of the sheet, as in the foregoing. As a result, the sheet will deform elastically in the inlet zone, where the pressure rises gradually until the flow criterion is reached and plastic deformation occurs.

Owing to this elastic deformation, the sheet will "round in" somewhat, which results in a "smoother" inlet geometry. This deformation will probably be of the same order of magnitude as the film thickness at the entrance. Hence it can be expected that this will lead to a significant increase in film thickness.

Aggarwal and Wilson [21] were the first to discuss the effect of workpiece rounding on film thickness. They extended the work of Wilson and Murch [22], who incorporated thermal effects into Wilson and Walowit's [9] work by allowing temperature differences between roll and sheet. Aggarwal and Wilson compared their calculated film thicknesses with experimentally obtained values from Azushima [23] and showed that their theory underestimates the film thicknesses by a factor of about five, even when the rolling speeds are low and temperature effects can be neglected. They postulated that this effect was due to plastic deformation of the workpiece in the inlet zone, the so-called "rounding in" phenomenon.

Tsao and Wilson [24] showed that this "rounding in" phenomenon does indeed lead to larger film thicknesses. They assumed the sheet to round in by local plastic deformation on the surface before plastic bulk deformation. The inlet film thickness was calculated analytically as a function of the workpiece radius and it was shown that this effect might easily double the calculated inlet film thickness. They also performed experiments on a laboratory mill with fully lubricated strips. In these experiments rolling was stopped with the strip partially deformed and the strip removed by reversing the mill. The strip was then sectioned on a plane normal to the roll axis during rolling and examined under a $250 \times$ optical microscope. No appreciable rounding of the workpiece in the inlet zone was visible. 
The present work will show that an increase of film thickness can indeed be explained by this rounding in phenomenon. However, this effect may not be due to plastic deformation but to elastic deformation of the workpiece. This also explains why Tsao and Wilson could not observe this phenomenon after their experiments.

An equation describing elastic deformation of the sheet can be derived by considering the force balance on a slab taken from the sheet. The derivation is similar to Von Kármán's equation, as described before. The only difference is that Von Mises' flow criterion has been replaced by Hooke's law.

\subsection{Vertical equilibrium}

In Fig. 1 a slab taken from the sheet is drawn. The vertical force balance between pressure $p$ and shear stress $\tau$ gives

$\sigma_{\mathrm{z}}=-p+\tau \frac{\mathrm{d} y}{\mathrm{~d} x}=-p\left(1-\mu \frac{d y}{\mathrm{~d} x}\right)$

where $\mu$ is the local coefficient of friction defined as $\mu=\tau / p$. (Note that $y$ is the magnitude of half the strip thickness, whereas a subscript $y$, as in $\epsilon_{y}$ and $\sigma_{y}$, denotes the direction along the rolls.) The coefficient of friction and the slope of the sheet in the contact zone are small compared to 1 , so their product can be neglected and the vertical force balance reads: $\sigma_{\mathrm{z}}=-p$.

\subsection{Horizontal equilibrium}

The horizontal force balance results in:

$\left(\sigma_{\mathrm{x}}+p\right) \frac{\mathrm{d} y}{\mathrm{~d} x}+y \frac{\mathrm{d} \sigma_{\mathrm{x}}}{\mathrm{d} x}+\tau=0$

\subsection{Shear stress}

In the work zone, the pressure flow can be neglected because of low pressure gradients and high viscosities. In the inlet zone, however, the pressure gradient is very large, resulting in a substantial contribution of lubricant back flow to the viscous shear stress. As a result, the shear stress must contain both Couette and Poiseuille flow contributions:

$$
\begin{aligned}
\tau & =-\frac{h}{2} \frac{\mathrm{d} p}{\mathrm{~d} x}+\eta \frac{\left(u_{\mathrm{rd}} / 2-u(x)\right)}{h} \\
& =-\frac{h}{2} \frac{\mathrm{d} p}{\mathrm{~d} x}+\frac{\eta}{2 h}\left[u_{\mathrm{rd}}-\left(2 u_{1} y_{1} / y\right)\right]
\end{aligned}
$$

\subsection{Horizontal stress $\sigma_{x}$}

In the inlet zone, where the deformation is elastic, the relation between stress and strain is given by Hooke's law.
Again, a plane strain situation is assumed (hence $\epsilon_{\mathrm{y}}=0$ ), so from Hooke's law then follows:

$\sigma_{\mathrm{y}}=\nu_{\mathrm{s}}\left(\sigma_{\mathrm{x}}+\sigma_{\mathrm{z}}\right)$

Introduction of $\epsilon_{\mathrm{z}}=\ln \left(y / y_{1}\right)$ and substitution of the above equations into the horizontal force balance (13) results in:

$$
\begin{aligned}
& {\left[\frac{-E_{\mathrm{s}}}{\nu_{\mathrm{s}}\left(1+\nu_{\mathrm{s}}\right)}\left(1+\ln \frac{y}{y_{1}}\right)-\frac{\left(1-2 \nu_{\mathrm{s}}\right)}{\nu_{\mathrm{s}}} p\right] \frac{\mathrm{d} y}{\mathrm{~d} x}} \\
& -\left(\frac{\left(1-\nu_{\mathrm{s}}\right)}{\nu_{\mathrm{s}}} y+\frac{h}{2}\right) \frac{\mathrm{d} p}{\mathrm{~d} x}+\frac{\eta}{2 h}\left(u_{\mathrm{rd}}-\frac{2 u_{1} y_{1}}{y}\right)=0
\end{aligned}
$$

This equation can be simplified by assuming the elastic strains to be small. Then $y / y_{1} \sim 1$ and so $1+\ln (y /$ $\left.y_{1}\right) \sim y / y_{1}$.

As a result, eqn. (15) is reduced to:

$$
\begin{aligned}
\frac{\mathrm{d} p}{\mathrm{~d} x}= & \left(\frac{2 \nu_{\mathrm{s}} y}{\nu_{\mathrm{s}} h+2\left(1-\nu_{\mathrm{s}}\right) y}\right)\left[\left(\frac{-E_{\mathrm{s}}}{\nu_{\mathrm{s}}\left(1+\nu_{\mathrm{s}}\right) y_{1}}-\frac{1-2 \nu_{\mathrm{s}}}{\nu_{\mathrm{s}}} \frac{p}{y}\right)\right. \\
& \left.\times \frac{\mathrm{d} y}{\mathrm{~d} x}+\frac{1}{y} \frac{\eta}{2 h}\left(u_{\mathrm{rd}}-2 \frac{u_{1} y_{1}}{y}\right)\right]
\end{aligned}
$$

This equation will be called the elastic Von Kármán equation.

\section{Flow stress}

To study the justification of the constant flow stress assumption (perfectly plastic material) it can be replaced by a relation described by Van der Lugt [25].

The flow stress is considered to consist of a static and a dynamic part. The static part is a function of strain, while the dynamic part is a function of strain rate and temperature. The temperature is calculated by assuming the heat to be generated by plastic deformation.

$\sigma_{\mathrm{u}}=\sigma_{\mathrm{st}}(\boldsymbol{\epsilon})+\sigma_{\mathrm{dn}}(\dot{\epsilon}, T)$

where $\sigma_{\mathrm{u}}$ is the flow stress from the uniaxial tension test. The plane strain flow stress $\sigma_{\mathrm{k}}$ is:

$$
\begin{aligned}
\sigma_{\mathrm{k}}(\epsilon, \dot{\epsilon}, T) & =\frac{2}{3^{1 / 2}} \sigma_{\mathrm{u}}(\epsilon, \dot{\epsilon}, T) \\
& =\frac{2}{3^{1 / 2}}\left[\sigma_{\mathrm{st}}(\epsilon)+\sigma_{\mathrm{dn}}(\dot{\epsilon}, T)\right]
\end{aligned}
$$

where

$$
\begin{aligned}
\sigma_{\mathrm{st}}(\epsilon)= & \sigma_{\mathrm{st} 0}+\alpha_{\mathrm{v}} G b \\
& \times\left[\frac{W}{\gamma+2 \beta_{\mathrm{s}}}\left(\mathrm{e}^{\left[(\gamma / 2)+\beta_{\mathrm{s}}\right] \epsilon}-1\right)+\left(\rho_{\mathrm{d} 0}\right)^{1 / 2}\right] \mathrm{e}^{-(\gamma / 2) \epsilon}
\end{aligned}
$$


and

$$
\sigma_{\mathrm{dn}}(\dot{\epsilon}, T)= \begin{cases}0 & \text { if } \dot{\epsilon} \leqslant \dot{\epsilon}_{\mathrm{s}} \equiv \dot{\epsilon}_{0} \mathrm{e}^{-\Delta G a k T} \\ \sigma_{\mathrm{dn} 0}\left[1+\frac{k T}{\Delta G_{0}} \ln \left(\frac{\dot{\epsilon}}{\dot{\epsilon}_{0}}\right)\right]^{q} & \text { if } \dot{\epsilon} \leqslant \dot{\epsilon} \leqslant \dot{\epsilon}_{0} \\ \sigma_{\mathrm{dn} 0} & \text { if } \dot{\epsilon} \geqslant \dot{\epsilon}_{0}\end{cases}
$$

Values for the different parameters in these equations, as given in the nomenclature list, are obtained from [25]. The plastic strain can be expressed in terms of the sheet thickness $y$ by subtracting the elastic strain, caused by workpiece rounding in the inlet, from the total strain:

$\epsilon=\epsilon_{\mathrm{tot}}-\epsilon_{\mathrm{e}}=-\ln \left(\frac{y}{y_{\mathrm{p} 0}}\right)+\ln \left(\frac{y\left(x_{\mathrm{s}}\right)}{y_{1}}\right)=-\left(\frac{y y_{1}}{y_{\mathrm{p} 0} y\left(x_{\mathrm{s}}\right)}\right)$

The strain rate is then

$\dot{\epsilon}=-u_{1} y_{1} \frac{1}{y^{2}} \frac{\mathrm{d} y}{\mathrm{~d} x}$

To calculate the temperature, a heat balance is formed, assuming an adiabatic situation and heat to be generated by plastic deformation only. This results in

$\frac{\mathrm{d} T}{\mathrm{~d} x}=-\frac{\sigma_{\mathrm{k}}}{y \rho C_{\mathrm{p}}} \frac{\mathrm{d} y}{\mathrm{~d} x}$

\section{Numerical aspects}

Again multigrid techniques have been used to solve the equations. In the inlet zone, the elastic Von Kármán equation (eqn. (16)), the Reynolds equation (eqn. (3)) and the film thickness equation (eqn. (5)) are solved. In the work zone the Von Kármán equation (eqn. (7)), the Reynolds equation, the film thickness, the temperature equation (eqn. (23)) and the flow stress equation (eqn. (18)) are solved. In the outlet zone the strip is assumed rigid and the film thickness and Reynolds equation are solved.

\section{Results}

Before presenting any results we would like to emphasize the assumed full film lubrication situation, implying perfectly smooth running surfaces. The results presented show film thicknesses which are smaller than the roughness of many rolled surfaces and thus may have little practical meaning. Nevertheless the calculations do give a general indication of the amount of real contact by comparison of the calculated film thicknesses with the average asperity height and provide a good starting point for mixed lubrication analysis. Also, the multigrid algorithm offers good possibilities to simulate surface roughness interaction.

First, a characteristic solution, solved using 49153 nodal points, will be presented. Next, the influence of the non-constant flow stress and elastic deformation of the sheet in the inlet on film thickness and pressure distribution will be studied.

Finally, a parameter study will be performed varying the inlet tension, the viscosity-pressure coefficient and the strip reduction.

Only the model problem is solved using a very large number of nodal points. The remaining calculations have been performed using 3073 nodal points in order to save CPU time and disk space.

The calculations have been restricted to steel rolling. In all calculations the start and the end of plastic deformation, $x_{\mathrm{s}}$ and $x_{e}$ respectively, are taken constant. As a result, all calculations show the same reduction and flow stress distribution.

\subsection{Model problem}

In Fig. 3(a) the solid curves represent the solutions for the pressure, the film thickness and the strip thickness (the dashed curve will be discussed later). This solution has been calculated using the input parameters from Table 2. The situation represents mediate gauge rolling of steel, lubricated by a Newtonian, low viscosity, mineral oil. The parameters with respect to the flow stress are given in the nomenclature list. The output parameters are presented in Table 3 .

It can be seen that in the inlet zone the pressure rises very steeply from zero until plastic deformation starts. As the film thickness is very small compared to the sheet thickness, the latter shows the parabolic roll shape. Just before the line joining roll centres $(x=0)$ the plastic deformation stops and the sheet becomes rigid.

The film thickness decreases very sharply in the inlet and remains almost constant in the work zone. Just before $x=0$ the film thickness shows a small dip, allowing a sharp drop of pressure.

The elastic deformation of the sheet in the inlet is very small compared with the plastic deformation, so it cannot be seen in Fig. 3(a). Therefore an enlargement around $x=x_{\mathrm{s}}$ is drawn in Fig. 3(b). Owing to the elastic deformation of the strip in the inlet zone, the strip "rounds in", making a larger lubricant flow possible. As a result the film thickness will be much larger than in the rigid situation. 

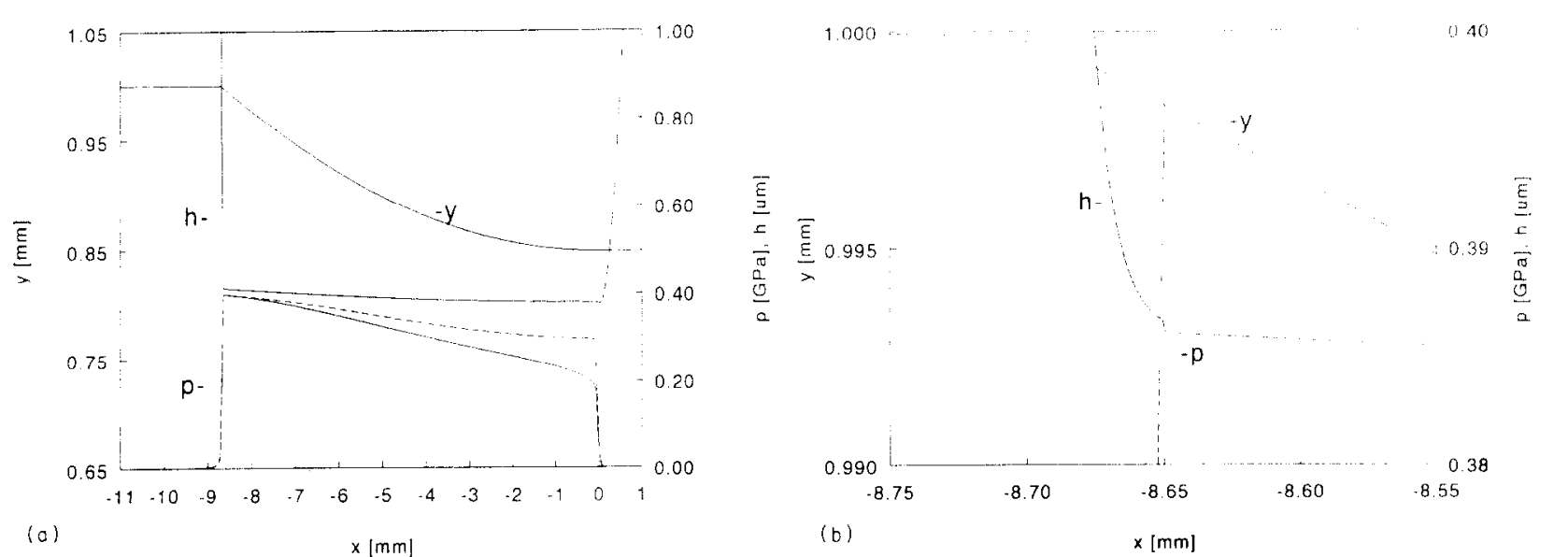

(a)

$x[\mathrm{~mm}]$
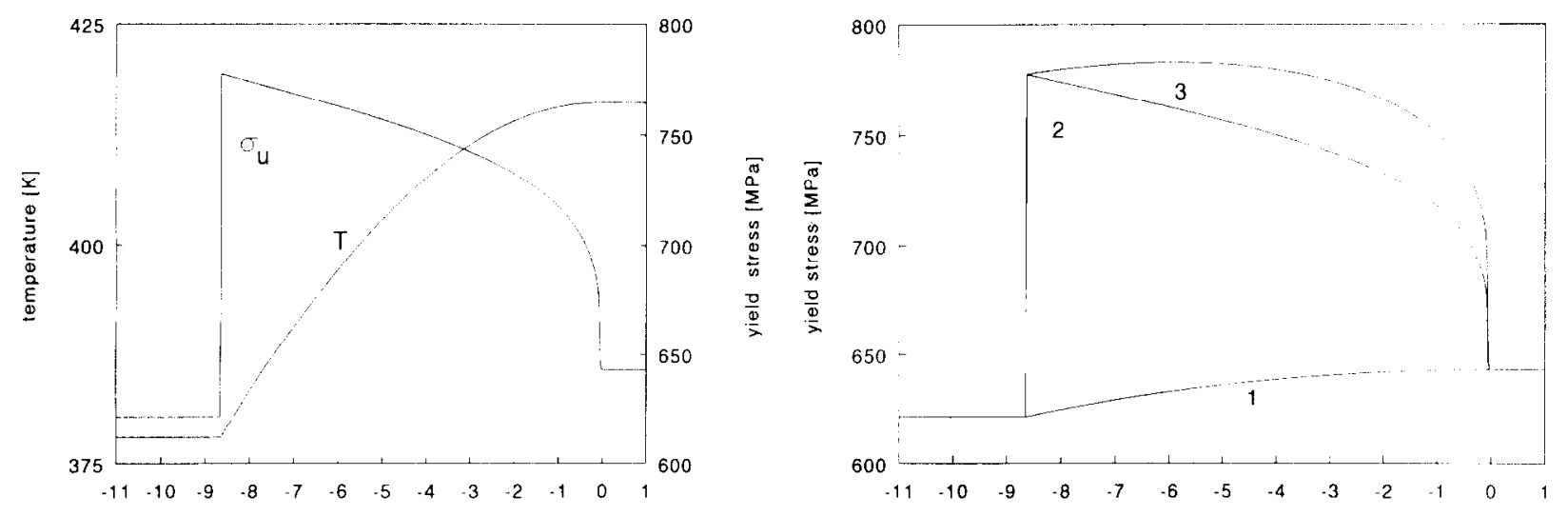

(c)

$x[\mathrm{~mm}]$

(d)

$$
x[\mathrm{~mm}]
$$

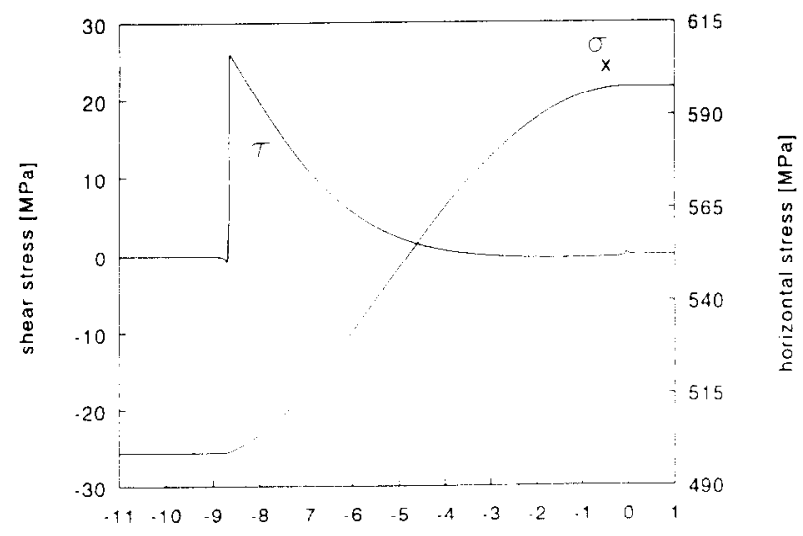

(e)

$x$ [mm]

Fig. 3. (a) Solution for the conditions given in Tables 2 and 3 showing half the sheet thickness $y$, pressure $p$ and film thickness $h$. The dashed line represents the pressure distribution when the flow stress is assumed to be constant. (b) Enlargement of (a) around the transition elastic-plastic deformation. (c) Temperature and flow stress for the same conditions as in (a). (d) Yield stress. $1=$ static component, $2=$ temperature dependent part of the flow stress, $3=$ flow stress for a constant temperature. (e) Shear stress and horizontal stress distribution. The parameters are the same as in (a).

Close to the elastic-plastic transition the film thickness shows an S-shape. This is due to the almost constant pressure gradient in this zone and the rather sharp change in pressure gradient at the point where plastic deformation starts. In Section 10.3 this will be discussed in detail.
In Fig. 3(c) the temperature and the flow stress are plotted. As mentioned before, heat generation is assumed to be caused by plastic deformation only, resulting in a temperature rise after $x=x_{\mathrm{s}}$. Since the strain rate decreases in the work zone the temperature gradient will also decrease. Finally, when the outlet zone is 
TABLE 2. Input parameters for the model problem. The parameters used with respect to the flow stress can be found in the Appendix

\begin{tabular}{lll}
\hline Parameter & Value & Dimension \\
\hline $\mathrm{n}$ & 49153 & - \\
$\eta_{\mathrm{p}}$ & 0.009 & $\mathrm{~Pa} \mathrm{~s}$ \\
$\alpha$ & $1.96 \times 10^{-8}$ & $\mathrm{~Pa}^{-1}$ \\
$\mathrm{u}_{\mathrm{rd}}$ & 20 & $\mathrm{~m} \mathrm{~s}^{-1}$ \\
$\mathrm{u}_{1}$ & 8.7 & $\mathrm{~m} \mathrm{~s}$ \\
$R$ & 0.25 & $\mathrm{~m}$ \\
$y_{\mathrm{s}}$ & $1.0 \times 10^{-3}$ & $\mathrm{~m}$ \\
$y_{p 0}$ & $2.0 \times 10^{-3}$ & $\mathrm{~m}$ \\
$\sigma_{\mathrm{i}}$ & 500.0 & $\mathrm{MPa}$ \\
$E_{\mathrm{s}}$ & 210.0 & $\mathrm{GPa}$ \\
$\nu_{\mathrm{s}}$ & 0.3 & - \\
$T_{1}$ & 378.0 & $\mathrm{~K}$ \\
$x_{\mathrm{s}}$ & -8.65 & $\mathrm{~mm}$ \\
$x_{\mathrm{e}}$ & -0.05 & $\mathrm{~mm}$ \\
\hline
\end{tabular}

TABLE 3. Output from the model problem

\begin{tabular}{lll}
\hline Parameter & Value & Dimension \\
\hline$w$ & 2674.2 & $\mathrm{kN} \mathrm{m} \mathrm{m}^{-1}$ \\
$h_{\mathrm{i}}$ & 0.3863 & $\mu \mathrm{m}$ \\
$h_{\min }$ & 0.3464 & $\mu \mathrm{m}$ \\
$y_{\mathrm{e}}$ & 0.8439 & $\mathrm{~mm}$ \\
$\left(y_{\mathrm{s}}-y_{\mathrm{e}}\right) y_{\mathrm{l}}$ & 15.133 & $\%$ \\
$\sigma_{\mathrm{u}}\left(x_{\mathrm{s}}\right)$ & 776.48 & $\mathrm{MPa}$ \\
$\sigma_{\mathrm{u}}\left(x_{\mathrm{e}}\right)$ & 643.11 & $\mathrm{MPa}$ \\
$\sigma_{\mathrm{o}}$ & 597.70 & $\mathrm{MPa}$ \\
$T_{\mathrm{e}}$ & 416.19 & $\mathrm{~K}$ \\
$x_{\mathrm{np}}$ & -3.27 & $\mathrm{~mm}$ \\
$\mu_{\max }$ & 0.066 & - \\
$p_{\max }$ & 0.399 & $\mathrm{GPa}$ \\
$\tau_{\max }$ & 26.15 & $\mathrm{MPa}$ \\
\hline
\end{tabular}

entered and the strain rate has become zero, the temperature has reached its maximum.

In Fig. 3(d) the total flow stress (2) and its static component (1) are plotted. The figure shows that the dynamic component causes the flow stress to decrease towards the outlet zone, partly due to the temperature rise in the contact. The figure also shows that heat development does have a large influence on the flow stress.

In Fig. 3(e) the shear stress and horizontal stress distributions are plotted. Owing to the non-constant flow stress, the pressures behind the neutral point are lower than in front of it. Consequently, the shear stress becomes very small here. Figure 3(e) shows a constant increase of the horizontal stress in the contact due to a constant decrease of pressure.

\subsection{The influence of a non-constant flow stress}

To show the effect of the use of the flow stress eqn. (18) on film thickness and pressure the model problem has been calculated using both a constant and a nonconstant flow stress. The pressure distribution for a constant flow stress is represented by the dashed curve in Fig. 3(a). The (constant) flow stress equals the flow stress at $x=x_{\mathrm{s}}$ in the model problem, i.e. $\sigma_{\mathrm{u}}=776.5$ GPa. Figure 3(a) shows that, owing to the decrease of the flow stress in the work zone, the pressure in this zone also decreases, resulting in a very large outlet tension. The pressure decrease for the constant flow stress case is, in the work zone, less pronounced. Consequently the shear stresses are also lower.

The inlet film thickness is, for both cases, practically the same. This is due to the fact that the film thickness is determined mainly by the inlet zone. In this zone, plastic deformation has not yet started.

\subsection{Influence of elastic sheet deformation and flow stress on inlet film thickness}

The influence of elastic deformation on film thickness is most pronounced when the pressure-viscosity coefficient $\alpha$ equals zero.

Two situations will be compared in this section, one using a constant flow stress $\left(\sigma_{\mathrm{u}}=775.25 \mathrm{MPa}\right)$ and a rigid inlet geometry, and a situation where the flow stress formula (18) is used and elastic sheet deformation is included. Both situations have been calculated using 49153 nodal points.

TABLE 4. Influence of the viscosity-pressure coefficient on inlet film thickness $h_{\mathrm{i}}$, inlet film thickness according to Wilson and Walowit $h_{\mathrm{w}}$, minimum film thickness $h_{\min }$, maximum coefficient of friction $\mu_{\max }$, maximum coefficient of friction using Barus divided by the maximum coefficient of friction using Roelands, and specific rolling force $w$ (for all calculations the Barus relation has been used)

\begin{tabular}{|c|c|c|c|c|c|c|}
\hline $\begin{array}{l}\alpha \\
\left(\mathrm{Pa}^{-1}\right)\end{array}$ & $\begin{array}{l}h_{\mathrm{i}} \\
(\mu \mathrm{m})\end{array}$ & $\begin{array}{l}h_{\mathrm{w}} \\
(\mu \mathrm{m})\end{array}$ & $\begin{array}{l}h_{\min } \\
(\mu \mathrm{m})\end{array}$ & $\mu_{\max }$ & $\frac{\mu_{\mathrm{B}}}{\mu_{\mathrm{R}}}$ & $\begin{array}{l}w \\
\left(\mathrm{kN} \mathrm{m}^{-1}\right)\end{array}$ \\
\hline 0 & 0.151 & 0.0367 & 0.126 & $1.9 \times 10^{-4}$ & 1.0 & 2392 \\
\hline $0.490 \times 10^{-8}$ & 0.226 & 0.0834 & 0.196 & $9.1 \times 10^{-4}$ & 2.1 & 2372 \\
\hline $0.981 \times 10^{-8}$ & 0.292 & 0.1460 & 0.256 & $5.0 \times 10^{-3}$ & 3.3 & 2373 \\
\hline $1.471 \times 10^{-8}$ & 0.356 & 0.2153 & 0.317 & $2.9 \times 10^{-2}$ & 3.8 & 2364 \\
\hline $1.870 \times 10^{-8}$ & 0.408 & 0.2730 & 0.371 & $1.7 \times 10^{-1}$ & - & 4090 \\
\hline
\end{tabular}


The sheet thickness, the film thickness and the pressure around the elastic-plastic transition are plotted in Fig. 4. In both cases plastic deformation starts at $x_{\mathrm{s}}=-8.65 \mathrm{~mm}$. In the rigid inlet situation (dashed curves, subscript B) this is easy to see; the sheet thickness is constant in the inlet zone and the start of plastic deformation is characterized by a discontinuity in the sheet thickness gradient.

In the elastic-plastic analysis (solid curves, subscript A), where the sheet deforms elastically, the elastic-plastic transition is much smoother than in the rigid case. Owing to this workpiece rounding, the buckle in the film thickness disappears. Also, the pressure gradient is much smaller than in the rigid inlet situation. In the rigid situation the pressure gradient shows a discontinuity which, of course, can also be seen in the film

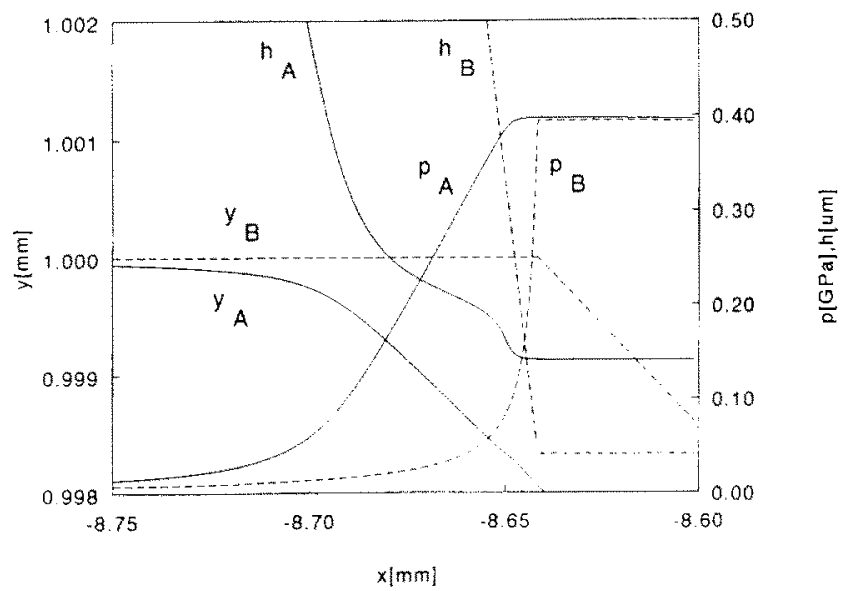

Fig. 4. Influence of elastic sheet deformation and non-constant flow stress on pressure $p$, sheet thickness $y$ and film thickness $h$ around the transition between the inlet and the work zone. The solid curves and subscript $A$ refer to the case where the sheet deforms elastically and the flow stress is non-constant. The subscript $B$ and the dashed curves refer to the case where the inlet geometry is assumed to be rigid and the flow stress is assumed to be constant. thickness. Owing to the dynamic component in the flow stress and the elastic deformation of the sheet, the pressure and film thickness round off near $x=x_{5}$.

In the rigid inlet and constant flow stress situation, the inlet film thickness is $0.041 \mu \mathrm{m}$, while in the elastic deformation and non-constant flow stress case it is 0.16 $\mu \mathrm{m}$. Hence, these effects have a significant effect on film thickness and could very well explain the discrepancy between the calculations and experiments as described in the introduction. Moreover, when developing mixed lubrication models these effects should be included.

\subsection{Varying the inlet tension}

In this section the inlet tension is varied, while the remaining input parameters are the same as in the model problem (Table 2). The inlet tension is varied from $450 \mathrm{MPa}$ up to $600 \mathrm{MPa}$ in steps of $50 \mathrm{MPa}$.

Varying the inlet tension results in a varying inlet pressure. Since the viscosity is exponentially dependent on pressure, larger pressures result in larger shear stresses. This is shown in Fig. 5(a). Owing to this increase in shear stress a "pressure hill" gradually appears, as can be seen in Fig. 5(b), resulting in a decreasing outlet tension. The inlet film thickness is almost independent of the inlet tension.

\subsection{Varying the viscosity-pressure coefficient}

The viscosity-pressure coefficient of the lubricant has a large influence on the viscosity and so on the film thickness, the viscous shear stress and the pressure. In this section the Barus viscosity-pressure relation is used to show the influence of the coefficient $\alpha$. When applying Roelands' relation the effect is less pronounced, but the trend remains the same.

An increase of $\alpha$ causes an almost linear increase of film thickness. This would cause a decrease of the shear stress, being inversely proportional to the film thickness. However, owing to the exponential viscos-

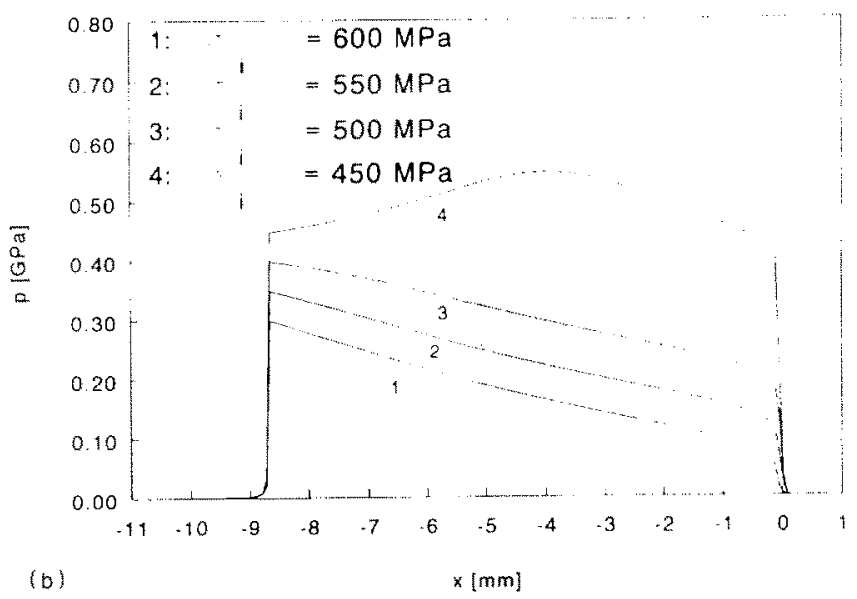

(a)

$x[\mathrm{~mm}]$

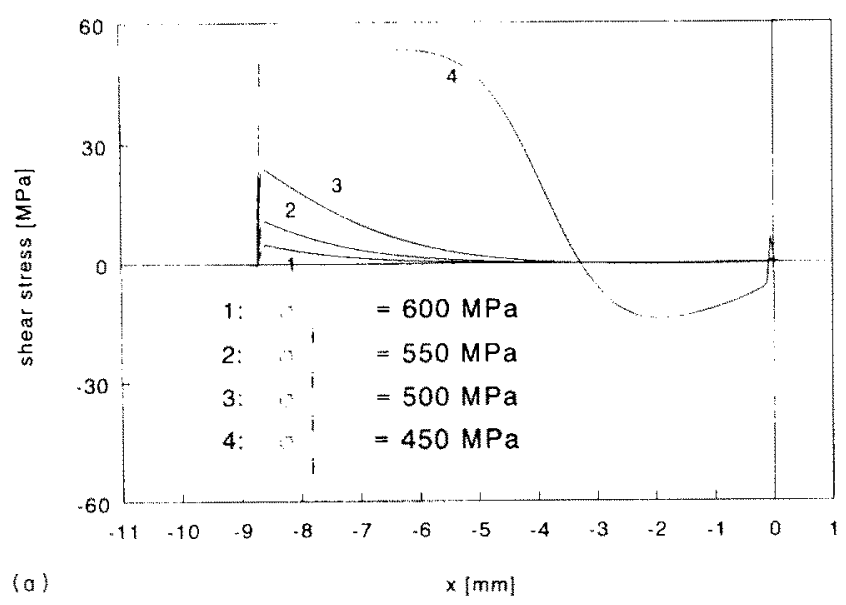

Fig. 5. (a) Shear stress distribution with varying inlet tensions.

(b) Pressure distribution with varying inlet tension. 


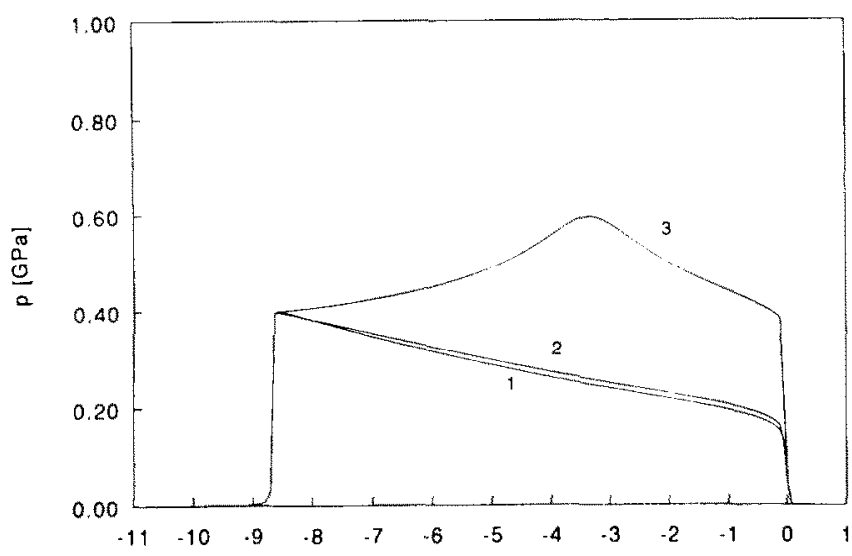

(a)

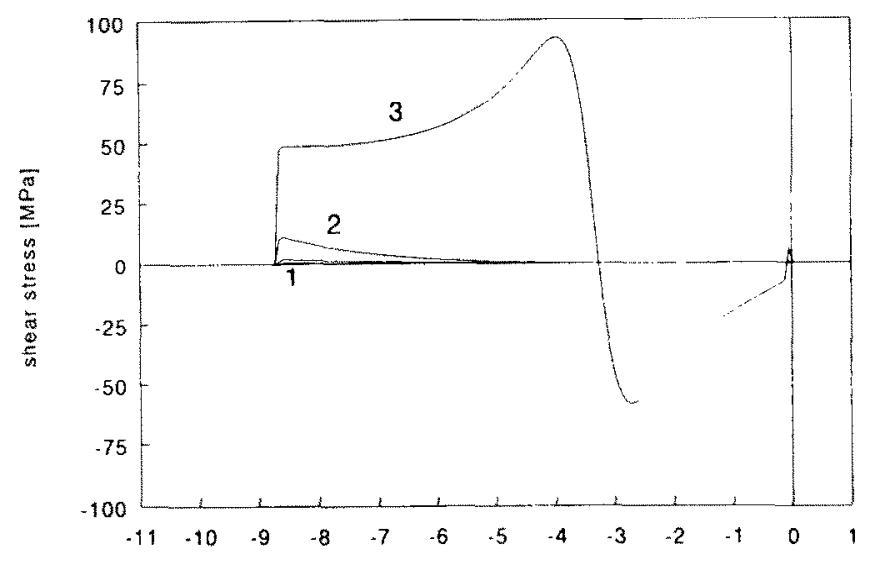

(b)

(b) The influence of $\alpha$ on the shear stress distribution. $1: \alpha=0 ; 2: \alpha=1.471 \times 10^{-8} \mathrm{~Pa}^{-1} ; 3: \alpha=1.870 \times 10^{-8} \mathrm{~Pa}^{-1}$.

ity-pressure relation, this latter effect will dominate. As a result there will be a small influence on shear stress distribution for lower values of $\alpha$. However, for higher values the shear stress strongly increases and so the pressure increases. This can be seen from Figs. 6 (a) and (b) respectively. Comparing $h_{\mathrm{w}}$ with $h_{\mathrm{i}}$ (Table 4) shows the large influence of elastic deformation.

\section{Conclusions}

Since the elastic deformation of the sheet in the inlet zone is large compared with the film thickness, the effect of this deformation has a large impact on the calculated film thickness, compared with the situation of pure plastic behaviour. This not only applies to steel rolling but also to aluminium rolling, where both pressure and Young's modulus are relatively small. This elastic strip deformation could very well be the effect Aggarwal and Wilson were looking for, as described in Section 7.

The flow stress behaviour has little or no influence on film thickness. However, it has a large influence on the pressure and shear stress distribution in the work zone.

A "pressure hill" appears at higher values of the viscosity-pressure coefficient. However, if the more realistic Roelands' relation is used the pressure distribution flattens again. Consequently, roughness effects must be incorporated when predicting friction in cold rolling.

The model provides a basis for the investigation of mixed lubrication effects. Owing to the use of multigrid techniques the computing time is restricted to $O(n)$, where $n$ is the number of nodal points used. This will be very beneficial when surface roughness effects are included, whenever a transient problem has to be solved requiring a fast and accurate solver. The influence of surface roughness on traction, pressure and film thickness in cold rolling is the next subject of investigation. The results will be presented in due time.

\section{Acknowledgements}

The authors would like to thank Hoogovens, IJmuiden, the Netherlands and Alcoa, Alcoa Center, USA for their financial support. Also the authors would like to thank Dr L. Devenpeck and Dr S. Sheu from Alcoa and Ing. R. L. Huisman and Dr J. van der Lugt from Hoogovens for their suggestions and helpful discussions.

\section{Appendix: Nomenclature}

$b \quad$ Burger's vector, $b, 2.5 \times 10^{-13} \mathrm{~m}$

$C_{\mathrm{p}} \quad$ specific heat sheet material, $C_{\mathrm{p}}=475.0 \mathrm{~N} \mathrm{m \times}$ $\mathrm{kg}^{-1} \mathrm{~K}^{-1}$

$d_{\mathrm{p}} \quad$ plastic deformation

$G$ shear modulus, $G=78.0 \mathrm{GPa}$

$h \quad$ film thickness

$h_{0} \quad$ constant

$h_{00} \quad$ constant

$h_{\mathbf{i}} \quad$ inlet film thickness

$h_{\min } \quad$ minimum film thickness

$h_{\mathrm{w}} \quad$ Wilson and Walowit inlet film thickness

$k \quad$ Boltzmann's constant, $k=8.617 \times 10^{-5} \mathrm{eVX}$ $\mathrm{K}^{-1}$

$n \quad$ number of nodal points

$p \quad$ pressure

$p_{\max } \quad$ maximum pressure

$p_{\mathrm{r}} \quad$ constant in Roelands equation $p_{\mathrm{r}}=1.962 \times$ $10^{8} \mathrm{~Pa}$

$q$ constant related to the dynamic part of the stress, $q=3.09$

$R \quad$ radius of roll

$T \quad$ strip temperature (K) 
$T_{1} \quad$ initial temperature, $T_{1}=378.0 \mathrm{~K}$

$T_{\mathrm{c}} \quad$ temperature at $x=x_{\mathrm{c}}$

$u \quad$ local strip surface velocity

$u_{1} \quad$ initial strip surface velocity

$u_{2} \quad$ outlet strip surface velocity

$u_{\mathrm{a}} \quad$ average velocity

$u_{\mathrm{r}} \quad$ peripheral roll velocity

$u_{\mathrm{rd}} \quad$ double peripheral roll velocity, $u_{\mathrm{rd}}=2 u_{\mathrm{r}}$

$w \quad$ load per unit width

$W \quad$ parameter related to dislocation, $W=2.724 \times$ $10^{11} \mathrm{~m}^{-1}$

$\sigma_{\mathrm{i}}$

$\sigma_{\mathrm{k}}$

$\sigma_{\mathrm{o}}$

$\sigma_{\mathrm{x}}$

$\sigma_{\mathrm{u}}$

$\sigma_{\text {st }}$

$\sigma_{\mathrm{dn}}$

$\sigma_{\mathrm{x}, \mathrm{y}, \mathrm{z}}$

$\sigma_{\mathrm{dn} 0}$

deformation energy production coordinate left boundary of the work zone right boundary of the work zone cavitation boundary location of neutral point half thickness of the strip inlet half thickness of the strip outlet half thickness of the strip half thickness after hot rolling, $y_{\mathrm{p} 0}=2.0 \times$ $10^{-3} \mathrm{~m}$ viscosity-pressure coefficient pressure coefficient of viscosity constant in flow stress equation, $\alpha_{v}=0.82$ parameter related to the ratio between apparent microscopic strain and macroscopic strain, $\beta_{\mathrm{s}}=0.174$

maximum value of the activation free energy, $\Delta G_{0}=0.985 \mathrm{eV}$

strain total strain elastic strain strain rate maximum strain rate, $\dot{\epsilon}_{0}=3.95 \times 10^{8} \mathrm{~s}^{-1}$ parameter related to dislocation production, $\gamma=9.536$

coefficient of friction

maximum coefficient of friction in the work zone viscosity viscosity at ambient pressure and inlet temperature inlet tension plane strain flow stress outlet tension tensile stress yield stress from uniaxial tension test static part of flow stress dynamic part of flow stress stress maximum value of the dynamic equivalent stress $=1.085 \mathrm{GPa}$ $\sigma_{\mathrm{sto}} \quad$ initial value of the static equivalent stress, independent of the dislocation density, $\sigma_{\text {sto }}=137.88 \mathrm{MPa}$

$\rho \quad$ density (strip material), $\rho=7850 \mathrm{~kg} \times \mathrm{m}^{-3}$

$\rho_{\mathrm{do}} \quad$ initial dislocation density, $\rho_{\mathrm{d} 0}=2.0 \times 10^{19} \mathrm{~m}^{-2}$

$\tau \quad$ shear stress

$\tau_{\max } \quad$ maximum shear stress

$\nu \quad$ poisson's ratio

\section{References}

1 Th. Von Kármán, Beitrag zur Theorie des Walzvoryanges (Contribution to the Theory of Rolling), Vorträge der Dresdener Tagung, Band 5, Heft 2, 1925.

2 D. Jortner, J. F. Osterle and C. F. Zorowski, Int. J. Mech. Sci., 2 (1960) 179-194.

3 H. S. Cheng, in Friction and Lubrication in Metal Processing, ASME, New York, 1966, pp. 69-80.

4 D. S. Bedi and M. J. Hillier, Proc. Inst. Mech. Eng. London, 182 (1967) 153-160.

5 W. R. D. Wilson and S. M. Mahdavian, J. Lubr. Technol., 96 (4) (1974) 572-578.

6 T. A. Dow, J. W. Kannel and S. S. Bupara, J. Lubr. Technol., 97 (1) (1975) 4-13.

7 Y. H. Tsao and L. B. Sargent, Trans. ASLE, 21 (1976) 20-24.

8 A. G. Atkins, Int. J. Mech. Sci., 16 (1970) 1-19.

9 W. R. D. Wilson and J. A. Walowit, in Tribology Convention 1971, Institute of Mechanical Engineers, London, 1971, pp. 164-172.

10 S. Sheu, Ph.D. Thesis, Northwestern University, Evanston, IL, 1985.

11 M. P. F. Sutcliffe, Proc. Inst. Mech. Eng. London, 204 (1990) 249-261.

12 O. Reynolds, Philos. Trans. R. Soc. London, 177 (1886) 157-234.

13 P. Cosse and M. Economopoulos, CNRM Metall. Rep., 17 (1968) 15-32.

14 C. Barus, Am. J. Sci., 45 (1893) 87-96.

15 W. E. Ten Napel, H. Moes and R. Bosma, Proc. Inst. Mech. Eng. London, 185 (1971) 37-71.

16 C. J. A. Roelands, Ph.D. Thesis, Technische Hogeschool Delft, The Netherlands, 1966.

17 A. A. Lubrecht, Ph.D. Thesis, University of Twente, Enschede, 1987.

18 C. H. Venner, W. E. ten Napel and R. Bosma, J. Lubr. Technol., 112 (1990) 426-432.

19 W. L. Briggs, A Multigrid Tutorial, SIAM, Philadelphia, P^, 1987.

20 A. Brandt, Multigrid Techniques: 1984 Guide with Applications to Fluid Dynamics, G.M.D., St Augustin 1, West Germany, 1984 (available as G.M.D.-Study No. 85, from G.M.D.-F1T, Postfach 1240, D-5205, St Augustin 1, Germany).

21 B. B. Aggarwal and W. R. D. Wilson, in Proc. 5th Leeds-Lyon Symposium on Tribology, Institute of Mechanical Engineers, London, 1978, pp. 351-359.

22 W. R. D. Wilson and L. E. Murch, J. Lubr. Technol., 98 (1976) 426-432.

23 A. Azushima, in Proc. First Int. Conf. on Lubrication Challenges in Metalworking and Processing, I.I.T.R.I., Chicago, IL, 1978, pp. 81-87.

24 P. Tsao and W. R. D. Wilson, in Proc. Int. Conf. Steel Rolling, Iron and Steel Institute of Japan, Tokyo, 1980, pp. 1143-1157.

25 J. van der Lugt, Ph.D. Thesis, University of Twente, Enschede, The Netherlands, 1988. 